\title{
Incremental value of volumetric quantification for myocardial perfusion imaging by computed tomography
}

\author{
Anna Oleksiak ${ }^{1 *}$, Cezary Kępka² ${ }^{2 *}$ Koen Nieman ${ }^{3}$, Mariusz Dębski ${ }^{2}$, Marcin Demkow ${ }^{2}$, Mariusz Kruk² \\ 'Department of Intensive Cardiac Therapy, National Institute of Cardiology, Warszawa, Poland \\ ${ }^{2}$ Department of Coronary and Structural Heart Diseases, National Institute of Cardiology, Warszawa, Poland \\ ${ }^{3}$ Departments of Cardiovascular Medicine and Radiology, Stanford University, School of Medicine, Stanford, CA, USA \\ *Both authors equally contributed to the study
}

Correspondence to:

Anna Oleksiak, MD, PhD,

Department of Intensive Cardiac

Therapy,

National Institute of Cardiology,

Alpejska 42, 04-628 Warszawa,

Poland,

phone: +482234343 14,

e-mail: aoleksiak@ikard.pl

Copyright by the Author(s), 2022

DOI: 10.33963/KP.a2022.0015

Received:

November 4, 2021

Accepted:

January 18, 2022

Early publication date:

January 18, 2022

\section{A B S T R A C T}

Background: The extent of myocardial ischemia is the crucial prognostic factor for interventional treatment decision making for coronary artery disease. The ability of computed tomography perfusion (CTP) to provide the missing volumetric information and its clinical value remains unknown.

Aims: The study aimed to compare a novel ischemic volume quantification method based on dynamic computed tomography perfusion (VOL CTP) with other CT-based imaging modalities for revascularization prediction.

Methods: In this prospective study, 53 (25 females, 63.5 [8.5] years old) consecutive symptomatic patients with $50 \%-90 \%$ coronary artery stenosis $(n \geq 1)$ on coronary computed tomography angiography underwent computed-tomography-derived fractional flow reserve (CT-FFR) analysis and dynamic CTP. We calculated the percentage of myocardial ischemia on the CTP-derived images. A 10\% cut-off was used to define functionally significant ischemia. The outcomes include coronary revascularization during the follow-up of 2.5 (interquartile range, 1.4-2.8) years. Physicians were blinded to the results of CTP and CT-FFR.

Results: Of the 53 patients in the study (68 arteries with 50\%-90\% stenosis), 16 underwent revascularization (12 elective, 4 event-driven). In the CTP quantitative analysis, 26 patients had ischemia. Overall, 18 patients had ischemia $\geq 10 \%$ on volumetric ischemia quantification based on dynamic computed tomography perfusion (VOL CTP), and 28 patients had CT-FFR $<0.8$. VOL CTP, standard CTP, CT-FFR, and computed tomography coronary angiography (CTA) $\geq 70 \%$ performed well for the prediction of total revascularization. Area under the curve was 0.973 vs. 0.865 , vs. 0.793 , vs. 0.668 , respectively. The VOL CTP with $\geq 10 \%$ cut-off was superior to the CT-FFR, standard CTP, and CTA $\geq 70 \%(P<0.001 ; P=0.002$ and $P<0.001$ respectively $)$.

Conclusions: VOL CTP quantification is feasible and adds important, actionable information to that provided by standard CTP or CT-FFR in patients with $50 \%-90 \%$ coronary artery stenosis.

Key words: computed tomography fractional flow reserve, computed tomography myocardial perfusion, dynamic computed tomography perfusion, myocardial ischemia

\section{INTRODUCTION}

Coronary computed tomography angiography (CTA) is recommended as the initial test for diagnosing coronary artery disease (CAD) in symptomatic patients in whom obstructive CAD cannot be excluded through clinical assessment alone [1]. Importantly, the anatomical assessment of the coronary arteries through anatomic methods is insufficient to predict functionally significant CAD in most (50\%-90\%) coronary artery stenosis cases [2]. To make appropriate decisions regarding further management, these patients usually require additional functional testing with regard to both the presence and the burden of ischemia. Recently, computed-tomography-derived fractional flow reserve (CT-FFR) and dynamic computed tomography perfu- 
WHAT'S NEW?

Our study delved into the untapped potential of dynamic computed tomography perfusion (CTP) examination and provided new information on the feasibility, optimal method, and clinical value of the additional volumetric myocardial ischemia assessment. Its main finding is that volumetric CTP adds incremental value to the traditional CTP or computed-tomography-derived fractional flow reserve (CT-FFR) in identifying patients requiring coronary revascularization in a long-term follow-up. According to our data, the new method may better facilitate decisions on what invasive therapies to use to improve the patient's prognosis. Our results underline the importance of ischemic volume quantification for the appropriate planning of interventional therapies in chronic coronary syndromes and allow for the identification of outpatients with a smaller ischemic area who may not benefit from revascularization.

sion (CTP) have been increasingly tested for the assessment of the functional significance of coronary stenosis [3-5]. Despite the suggested equivalence of the diagnostic values of CT-FFR and dynamic CTP, however, both imaging modalities have unique advantages and disadvantages [3-5]. CT-FFR requires a high-quality CTA scan and preferably a lower calcium score, whereas dynamic CTP needs additional radiation but is more robust in patients with compromised quality coronary CTA or high calcium loads [4, 5].

One of the seminal considerations in the functional assessment of CAD is the myocardial ischemic burden. The invasive therapies are endorsed particularly for patients with a large ischemic area defined as $>10 \%$ of the left ventricle (LV) because in such cases, coronary revascularization can improve the outcome [6]. CT-FFR does not offer such an assessment, but volumetric ischemia assessment may theoretically be done via CTP. Despite the potential added clinical value of volumetric perfusion deficits, the previous studies on CTP have not addressed this issue [7].

Therefore, our study aimed to develop a method for volumetric ischemia quantification based on dynamic CTP (VOL CTP) and evaluate its potential clinical impact in the context of CT-based anatomic and functional CAD diagnostic methods.

\section{METHODS}

\section{Study population and follow-up}

Patients who had undergone CTA due to suspected CAD and those with $\geq 150 \%-90 \%$ coronary artery stenosis were recruited for the ULYSSES study. The study exclusion criteria were symptoms of unstable coronary artery disease or acute coronary syndrome, the history of myocardial infarction, the history of percutaneous coronary intervention (PCl) or coronary artery bypass grafting, the glomerular filtration rate (GFR) $<60 \mathrm{ml} / \mathrm{min} / 1.72 \mathrm{~m}^{2}$, body mass index (BMI) $>35 \mathrm{~kg} / \mathrm{m}^{2}$, contraindications to computed tomography (including pregnancy, etc.); contraindications to the administration of an iodine contrast media or regadenoson; heart failure with reduced ejection fraction, a significant valvular heart disease, aortic aneurysm or aortic dissection; persistent atrial fibrillation or atrial flutter; hypertrophic cardiomyopathy. All patients had a 12-lead ECG before study examinations.

\section{Clinical outcomes}

Our study was part of the ULYSSES study (ClinicalTrials. gov NCT03917199). Caring physicians were blinded to the CTP and CT-FFR results. The patients were referred for invasive coronary angiography based on the clinical pathway recommended by the European Society of Cardiology (ESC) $[1,6]$. The elective revascularizations were ischemia-guided (non-invasive imaging including CMR or invasive FFR). All the elective therapeutic procedures were completed 6 months after the initial examination. In all the patients who underwent non-elective revascularization, either invasive FFR or the evidence of acute ischemia according to the ESC Fourth Universal Definition of Myocardial Infarction was used as appropriate to guide revascularization $[1,6,9]$.

Follow-up information was gathered through a review of the patients' hospital records and telephone interviews with the patients at a median of 2.5 (1.4-2.8) years from study enrolment. Elective and total (elective and event-driven) revascularizations were used as the study outcomes. No deaths were recorded.

The study protocol complied with the Declaration of Helsinki and received approval from the Ethics Committee at the National Institute of Cardiology. All the patients gave their written informed consent.

\section{Computed-tomography angiography protocol and analysis}

Coronary CTA was performed on a dual-source Somatom Force CT scanner (Siemens, Forchheim, Germany). Sublingual nitrates were administered before scanning in all the patients. If necessary, $\beta$-blockers were administered intravenously, targeting a heart rate of $<70 \mathrm{bpm}$. The protocol for CTA image acquisition was recommended to comply with the guidelines [10]. Assessment of luminal stenosis was performed by an experienced reader (MK/CK $>16$ years' experience with (TA) using an 18-segment coronary model with CT coronary application through the Syngo.via software (Siemens Medical Systems). The calcium score was calculated according to the Agatston method. For all the 


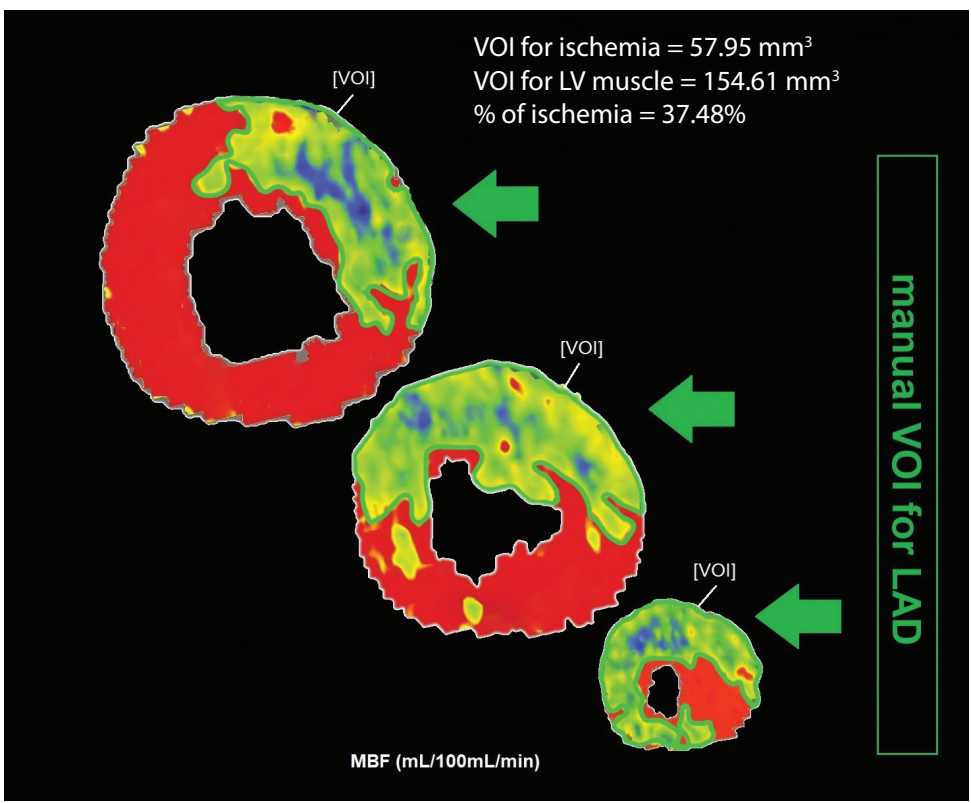

Figure 1. Methodology of volumetric perfusion analysis. Sample methodology of the volume of interest (volume of interest, green arrows) measurement at short-axis heart cross-sections (only selected ones are presented) and percentage of ischemia calculation patients, the CAD reporting and data system (CAD-RADS) was evaluated.

\section{Computed tomography-derived fractional flow reserve analysis}

CT-FFR calculations were performed on the coronary CTA datasets onsite using the CFFR version 3.2.0 research software (Siemens, Germany) with previously validated diagnostic performance [11-14]. The software allows the computation of CT-FFR values in selected locations of the coronary tree and displays the CT-FFR values along the vessel in the form of color-coded coronary artery filling.

Data preparation required acceptance or correction of the luminal center lines and contours automatically generated by the software. The observer then marked any stenotic lesions before the software generated a patient-specific, three-dimensional mesh of the coronary artery tree (MD) validated by a second observer (MK). All discrepancies were resolved by consensus. The simulated CT-FFR value was established by one observer (MK), who was blinded to the CTA and CTP results. The CT-FFR value was measured $40 \mathrm{~mm}$ distal to the minimal luminal area [13]. CT-FFR $<0.80$ was considered significant.

\section{Computed tomography perfusion protocol}

The recruited patients underwent dynamic CTP using a dual-source CT scanner (Somatom Force, Siemens, Germany). A detailed description of the CTP protocol was published previously [8].

\section{Computed tomography perfusion data analysis}

The anonymized CTP data were analyzed by an experienced reader $(A O,>10$ years' experience with CTA and $>5$ years' experience with (TP) in a core lab at the National Institute of Cardiology in Warsaw, Poland. The interpretation differences were resolved through consensus with the second experienced reader (MK/CK >16 years' experience with CTA and $>5$ years' with CTP). The readers were blinded to the clinical history of the patients and the CTA results. Semi-automatic analyses of the dynamic CTP images were done using commercial software (CT Myocardial Perfusion, Siemens, Germany). Motion correction was applied if needed to correct the breathing-related artifacts or artifacts due to extrasystole. The endocardial and epicardial contours of the left ventricle (LV) were segmented automatically, with manual correction if needed. The images were analyzed with a constant window width/level for all the patients.

The CTP standard analysis included the assessment of ischemia presence in a 16-segment model by measuring the circular region of interest (ROI). The ROI had a minimal area of $50 \mathrm{~mm}^{2}$. The indexed myocardial blood flow (index-MBF) with a $<0.78$ threshold was used to define myocardial ischemia [8]. The patient was diagnosed as having ischemia in CTP if the hypoperfusion involved at least one myocardial segment of an at-least-50\% subendocardial layer.

Volumetric analysis of ischemia (volume of interest [VOI] of ischemia, $\mathrm{mm}^{3}$ ) was done using manual VOI contours based on the index-MBF threshold on short-axis cross-sections for each slice. To normalize the inter-individual differences, the optimal patient-specific index-MBF was defined as the measured MBF/MBF (LV) $75 \%$ and was used at the 0.78 threshold for all the VOI measurements [8]. Therefore, the VOI contours included the area within the myocardial wall with index-MBF $<0.78$. In the next step, the measured $\mathrm{VOI}$ of ischemia was divided by the automatically measured total VOI for the LV muscle (VOI of LV muscle, $\mathrm{mm}^{3}$ ). An example is presented in Figure 1. Like other non-invasive imaging methods of ischemia detection, $10 \%$ ischemia was used to define functionally significant ischemia in VOL CTP. 
Table 1. Baseline patient characteristics

\begin{tabular}{|c|c|c|c|c|}
\hline Parameter & $\begin{array}{l}\text { Study group } \\
\qquad(\mathrm{n}=53)\end{array}$ & $\begin{array}{l}\text { Total revascularization } \\
\qquad(n=16)\end{array}$ & $\begin{array}{l}\text { No revascularization } \\
\qquad(n=37)\end{array}$ & $P$-value \\
\hline Age, years, mean (SD) & $62.5(8.5)$ & $58.8(9.0)$ & $65.9(7.5)$ & 0.4 \\
\hline Male sex, n (\%) & $28(53 \%)$ & $12(75 \%)$ & $16(43 \%)$ & 0.04 \\
\hline $\mathrm{BMI}, \mathrm{kg} / \mathrm{m}^{2}$, mean (SD) & $27.7(3.6)$ & $27.9(3.4)$ & $27.6(3.7)$ & 0.8 \\
\hline Height, cm, mean (SD) & $168.0(9.0)$ & $171.3(7.5)$ & $166.5(9.3)$ & 0.07 \\
\hline \multicolumn{5}{|l|}{ CAD risk factors } \\
\hline Hypertension, n (\%) & $46(87 \%)$ & $14(88 \%)$ & $32(86 \%)$ & 1.0 \\
\hline Dyslipidemia, n (\%) & $52(98 \%)$ & $16(100 \%)$ & $36(97 \%)$ & 1.0 \\
\hline Diabetes, n (\%) & $13(25 \%)$ & $3(19 \%)$ & $10(27 \%)$ & 0.7 \\
\hline CAD family history, $\mathrm{n}(\%)$ & $31(58 \%)$ & $10(63 \%)$ & $21(57 \%)$ & 0.7 \\
\hline Active smoking, n (\%) & $5(9 \%)$ & $4(25 \%)$ & $1(2.7 \%)$ & 0.02 \\
\hline Past smoking ${ }^{\mathrm{a}}, \mathrm{n}(\%)$ & $26(49 \%)$ & $10(63 \%)$ & $16(43 \%)$ & 0.2 \\
\hline
\end{tabular}

asmoking cessation $>1$ year ago

Values are presented as mean (SD) or $\mathrm{n}(\%)$

Abbreviations: $\mathrm{BMI}$, body mass index, $\mathrm{CAD}$, coronary artery disease

Table 2. Baseline computed tomography coronary angiography results

\begin{tabular}{l|c|c|c|}
\multicolumn{1}{c|}{ Parameter } & $\begin{array}{c}\text { Study group } \\
\mathbf{( n = 5 3 )}\end{array}$ & $\begin{array}{c}\text { Total revascularization } \\
(\mathbf{n = 1 6 )}\end{array}$ & $\begin{array}{c}\text { No revascularization } \\
\text { (n= 37) }\end{array}$ \\
\hline $\begin{array}{l}\text { CASC (Agatston) } \\
\text { One- or multi-vessel disease }\end{array}$ & $366.7(109.2-727.6)$ & $216.6(67.4-854.2)$ & $417.5(125.1-656.1)$ \\
3-vessel CAD & 0 & 0 & 0 \\
2-vessel CAD & $15(28 \%)$ & $7(44 \%)$ & $8(22 \%)$ \\
1-vessel CAD & $38(72 \%)$ & $9(56 \%)$ & $29(78 \%)$ \\
CAD-RADS & & & 0.1 \\
0-2 & 0 & 0 & 0 \\
3 & $41(77 \%)$ & $6(37 \%)$ & $35(95 \%)$ \\
4 A & $12(23 \%)$ & $10(63 \%)$ & $2(5 \%)$ \\
4B & 0 & 0 & 0 \\
5 & 0 & 0 & $<0.001$ \\
\hline
\end{tabular}

Values are $\mathrm{n}(\%)$ or median (interquartile range [IQR])

Abbreviations: CAD, coronary artery disease, CAD-RADS, Coronary Artery Disease - Reporting and Data System; CASC, coronary artery calcium score

The members of the recruiting team and the referring physicians were blinded to the CTP results. The patients' management was independent of the CTP results.

\section{Statistical analysis}

The continuous variables are presented herein as mean with standard deviation (SD) or median with interquartile range (IQR), as appropriate. The categorical variables are presented as frequencies and percentages. For the assignment of intra- and inter-observer variability, 20 anonymized CTP studies were re-analyzed by the same reader after 1 month, and by a second blinded reader (a standard approach using the MBF per segment and volumetrically as a percentage of ischemia per patient). The intra- and inter-observer variability were calculated using intraclass correlation coefficients (ICCs). Homogeneity analysis of variance for the variables with a normal distribution was done using Levene's test. The differences between the patients who underwent revascularization during the follow-up and those who did not were determined through Student's t-test (normal distribution) or the U Mann-Whitney test (non-normal distribution, independent variables). The differences between the qualitative variables were determined using the chi-square test or Fisher's exact test. In further analyses, a comparison of the receiver operating curves was used (elective or total revascularization as a classifier). The accuracy of the diagnostic methods was compared using McNemar's test. The net reclassification improvement (NRI) was calculated for VOL CTP in comparison to CTP standard analysis. $P<0.05$ was considered statistically significant. The analyses were performed using MedCalc (18.11.3, Ostend, Belgium).

\section{RESULTS}

\section{Baseline characteristics}

The study population consisted of 53 patients ( 28 males and 25 females, 63.5 [8.5] years old, 27.7 [3.6] kg/m²). There were no differences in age, $\mathrm{BMI}$, the prevalence of diabetes, hypertension, dyslipidemia, smoking in the past, family history of CAD, and medication use between the patients who underwent revascularization and those who did not. There were more males and current smokers in the revascularization group ( $P=0.04$ and $P=0.01$, respectively). The baseline characteristics of the study population are given in Table 1, Table 2, and Supplementary material, Table S1. 

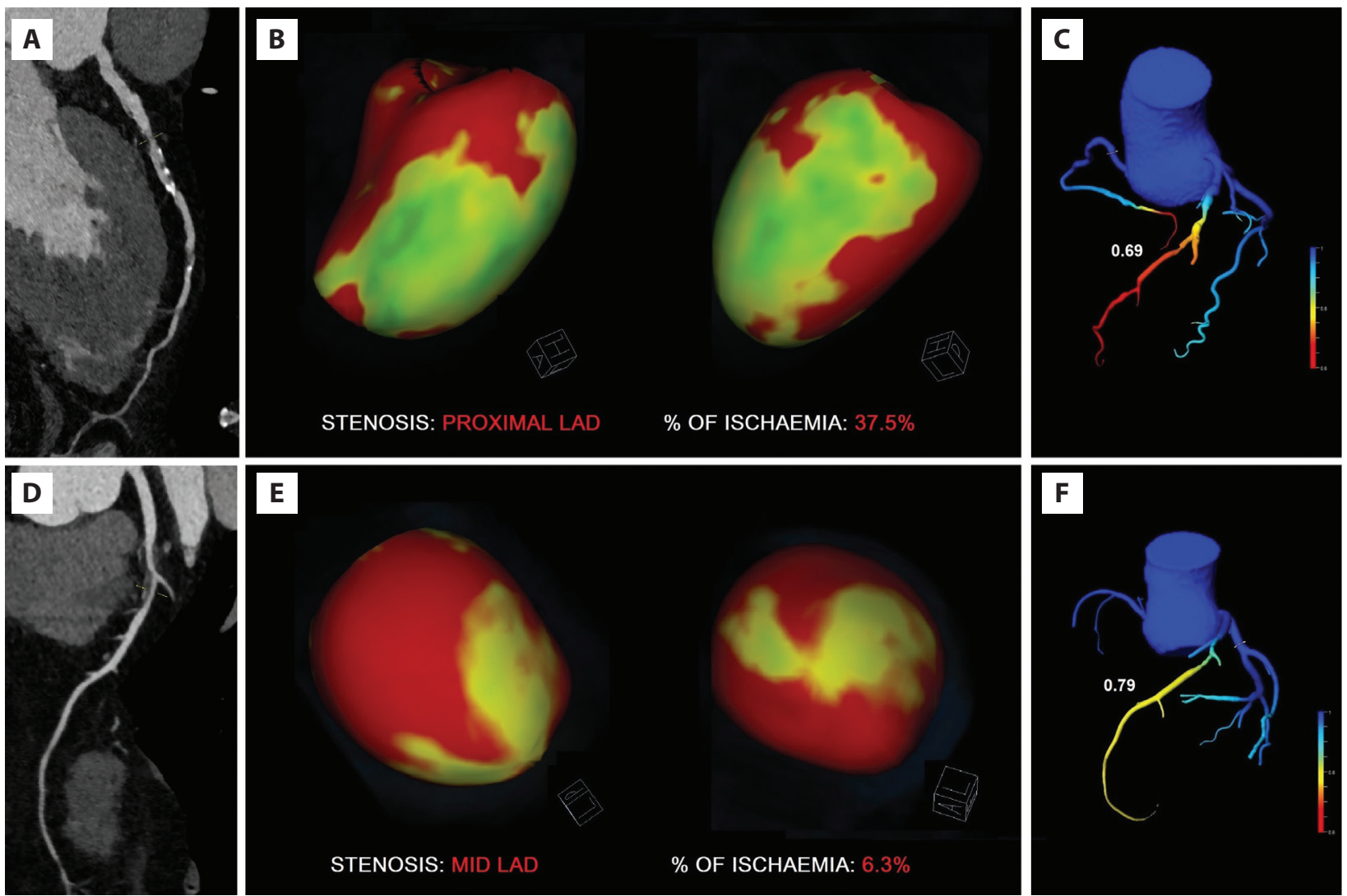

Figure 2. Results of coronary computed tomography angiography (CTA) (A, D), dynamic computed tomography perfusion (CTP) (B, E), and computed tomography-derived fractional flow reserve (CT-FFR) (C, F). 3D volume perfusion images of the left ventricle showing the ischemia extent and CT-FFR in different patients

\section{Study outcomes}

Twelve patients (22.6\%) underwent elective revascularization following the study procedures within 6 months since CTA, and four patients (7.5\%) underwent event-driven revascularization (1 non-fatal myocardial infarction, 3 unstable angina requiring hospitalization) during the 2.5-year (1.4-2.8) follow-up. Additionally, two patients had symptom progression during the follow-up and were referred by their physicians for invasive angiography (both had initially normal (TP). They underwent invasive angiography without revascularization (invasive FFR $>0.8$ in both cases).

\section{Imaging results and revascularization prediction}

In the 53 patients in the study, 159 coronary arteries were evaluated via CTA, and 68 (43\%) were found to be $50 \%-90 \%$ stenosed. There were no differences in coronary artery calcium (CASC) score. The baseline CTA findings are provided in Table 2.

Through CT-FFR analysis, ischemia was detected in 28 patients. CT-FFR was not performed in three patients (one of whom had elective revascularization) due to heavy calcifications.

In CTP examinations, all images had optimal quality and were interpretable. Intra-observer and inter-observer ICC's for MBF as a representative measure from $\mathrm{ROI}$ were excellent: 0.987 (0.982-0.990) and 0.985 (0.979-0.989), respectively. Intra-observer and inter-observer ICC's for volumetric CTP (measured as percentage of ischemia per patient) were excellent: 0.999 (0.999-1.000) and 0.998 (0.994-0.999), respectively. The time needed to postprocess a CTP data set with volumetric ischemia evaluation was 10-15 minutes.

The median radiation dose for topogram and lowdose non-contrast scan was 38.6 (36.6-39.6) $\mathrm{mGy} \times \mathrm{cm}$ (0.54 [0.51-0.55] mSv) and, for dynamic CTP scan, was 352.0 (276.4-496.6) mGy $\times$ cm (4.93 [3.87-6.95] mSv).

Through the CTP standard quantitative analysis, an ischemic territory involving at least one myocardial segment was detected in 26 (49\%) patients. According to the volumetric CTP analysis results, the mean ischemia percentage in the patients who underwent revascularization was $23.8(8.5) \%$ vs. $2.2(4.7) \%(P<0.001)$ in the non-revascularized patients. The sample results are presented in Figure 2. In total, the VOL CTP analysis revealed that 18 patients had over $10 \%$ ischemia, 16 of whom underwent revascularization ( 12 elective, 4 event-driven).

The clinical outcomes associated with different diagnostic strategies (CTA, CT-FFR, CTP, and VOL CTP-guided cohort) are summarized in Figure 3. The area under the curve (AUC) for the prediction of elective revascularizations by CTA $\geq 50 \%$ was 0.500 ( $95 \%$ confidence interval, $0.359-0.641$ ), by CTA $\geq 70 \%$ was 0.692 (95\% Cl, $0.550-0.812$ ), 


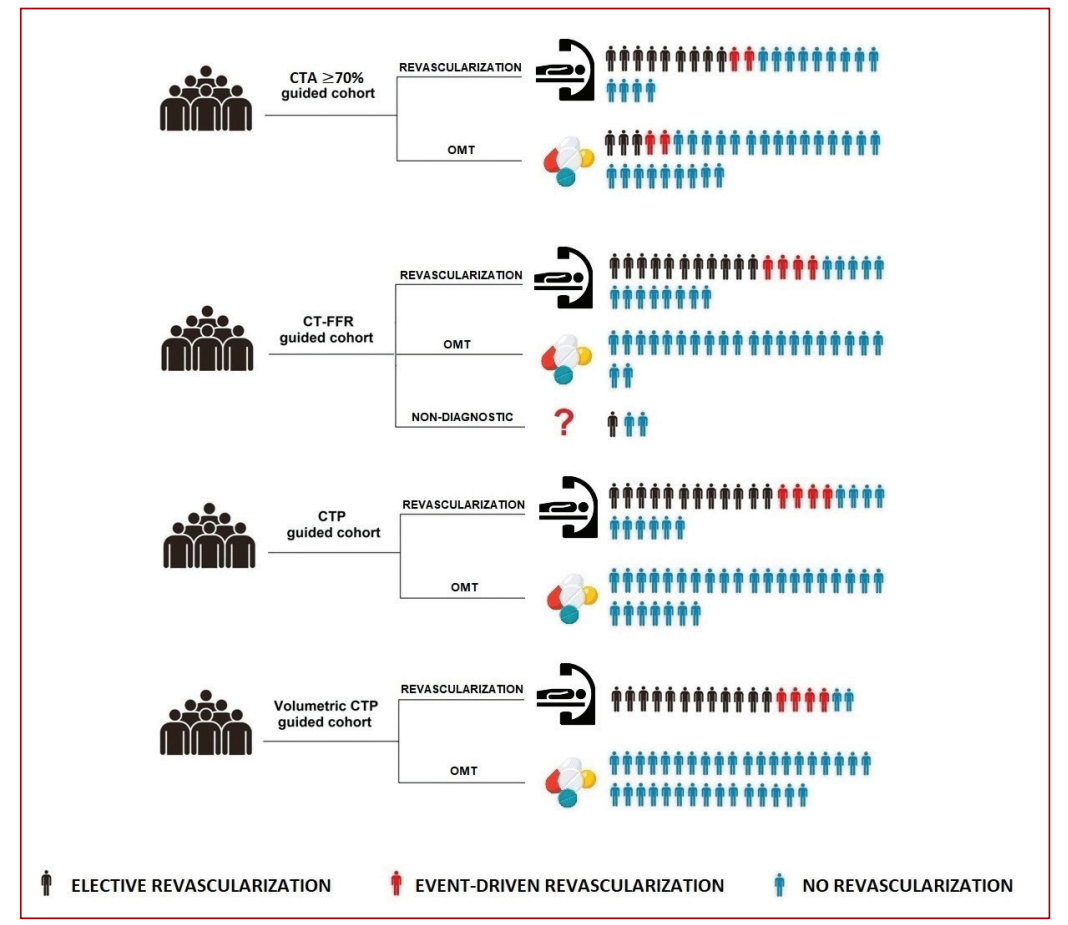

Figure 3. Summary of computed tomography-based diagnostic pathways with outcomes. Summary of the diagnostic pathways (CTA, CT-FFR, CTP, and volumetric CTP guided cohort) with outcomes (total revascularizations) Abbreviations: OMT, optimal medical treatment; other - see Figure 2
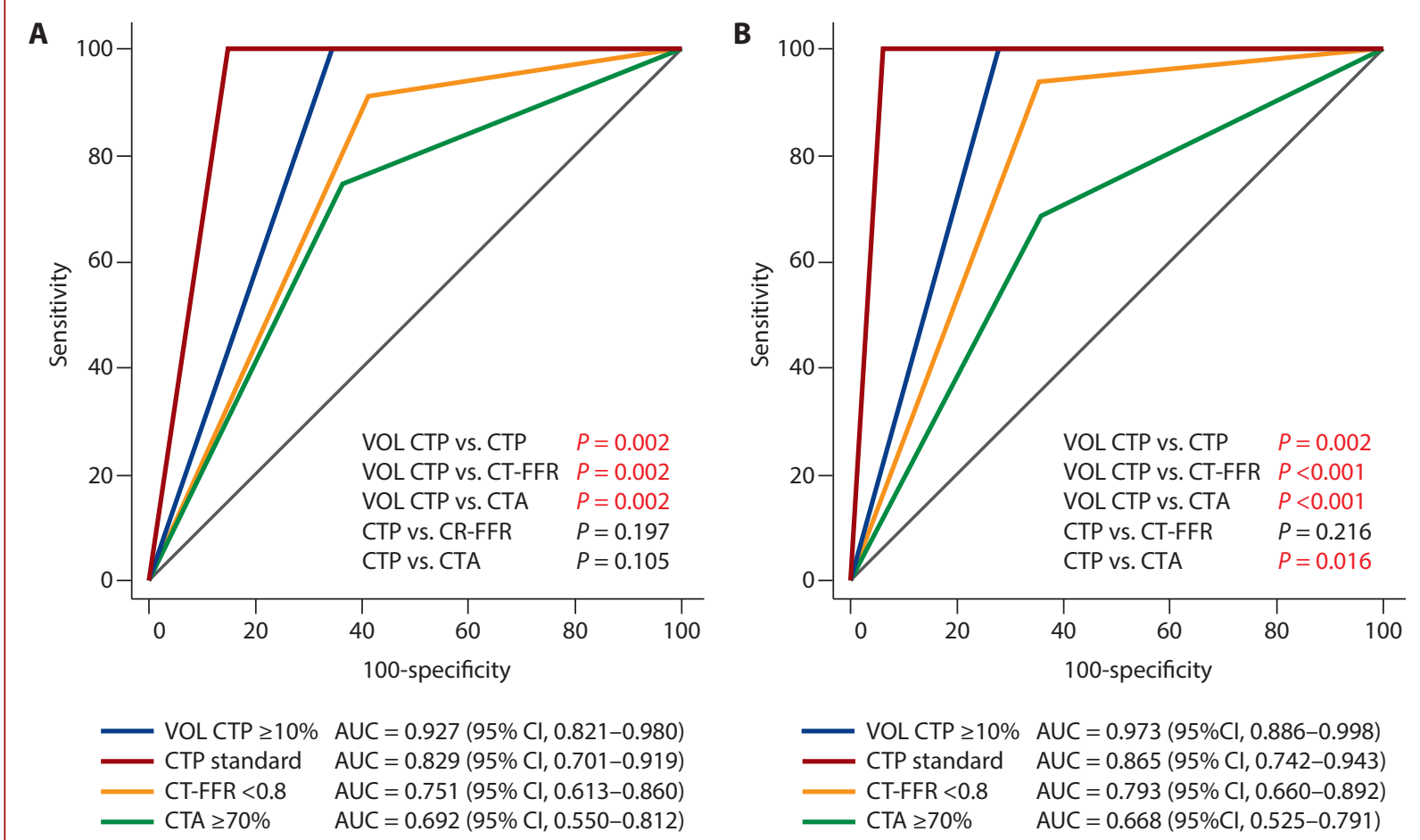

Figure 4. Comparison of ROC curves for revascularization prediction. Comparison of ROC curves for revascularization prediction (A - elective, $\mathbf{B}$ - total revascularizations) by CTA, CT-FFR, CTP using a standard approach and volumetric CTP (VOL CTP $\geq 10 \%)$

Abbreviations: see Figure 2

by CT-FFR was $0.751(95 \% \mathrm{Cl}, 0.613-0.860)$, by the CTP standard approach was $0.829(95 \% \mathrm{Cl}, 0.701-0.919)$, and by VOL CTP ( $\geq 10 \%$ ) was 0.927 ( $95 \% \mathrm{Cl}, 0.821-0.980)$. The ischemia detection via CTP using the standard approach was equal to that via CT-FFR for the prediction of elective revascularizations. The VOL CTP with a $\geq 10 \%$ cut-off, however, was better than the other methods in predicting elective revascularization (Figure $4 \mathrm{~A}$ ).

The AUC for the prediction of total revascularizations by CTA $\geq 50 \%$ was $0.500(95 \% \mathrm{Cl}, 0.359-0.641)$, by CTA $\geq 70 \%$ was $0.668(95 \% \mathrm{Cl}, 0.525-0.791)$, by CT-FFR was $0.793(95 \% \mathrm{Cl}, 0.660-0.892)$, by the CTP standard approach 


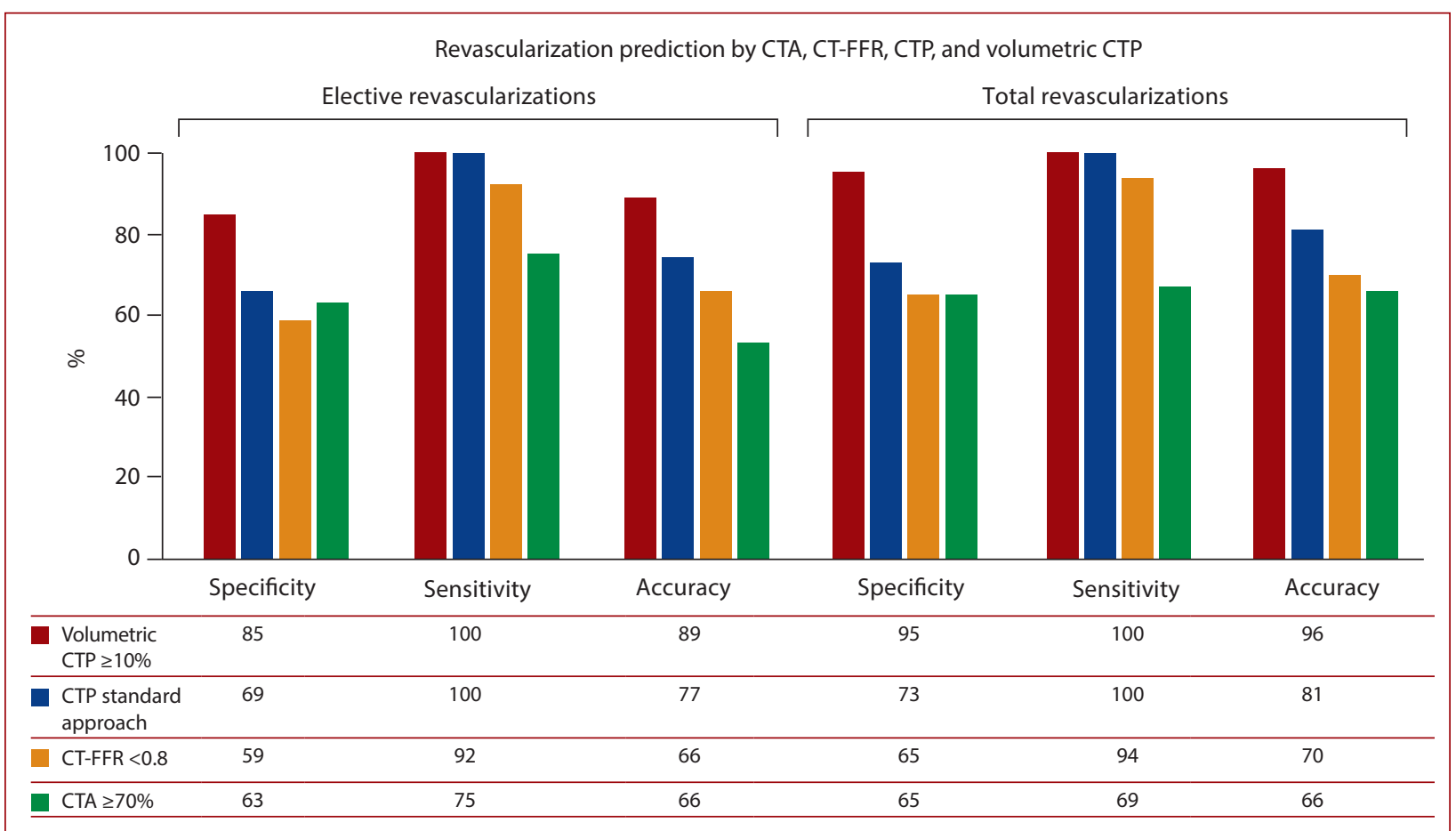

Figure 5. The diagnostic value of CT-based imaging for revascularization prediction.

Specificity, sensitivity, and accuracy of elective and total revascularization prediction during the follow-up by CTA, CT-FFR, CTP with a standard approach and volumetric CTP

Abbreviations: see Figure 2

was 0.865 (95\% Cl, 0.743-0.943), and by VOL CTP ( $\geq 10 \%)$ was 0.973 [95\%Cl: 0.886-0.998]. The ischemia detection via CTP using the standard approach was equal to that via CT-FFR for the prediction of total revascularizations, but the VOL CTP with $a \geq 10 \%$ cut-off was better than other methods in predicting total revascularization (Figure 4B).

The sensitivity, specificity, and accuracy of the analyzed imaging methods are presented in Figure 5 and Supplementary material, Table S2. The accuracy of VOL CTP $(\geq 10 \%)$ was better than CTP $(P=0.008)$, CT-FFR $(P=0.007)$, and CTA $\geq 70 \%(P<0.001)$ for total revascularization prediction.

The net reclassification index was 0.30 (30\%) for VOL CTP as compared to CTP with a standard approach for both elective and total revascularizations.

\section{DISCUSSION}

Our study delved into the untapped potential of dynamic CTP examination and provided new information on the feasibility, optimal method, and clinical value of the additional volumetric myocardial ischemia assessment. According to our data, the new method is applicable and diagnostically robust compared to the more traditional alternatives for the prediction of coronary revascularization in the longterm follow-up and may facilitate decision-making on the invasive therapies to use, accounting for the prognostic dimension. The added ability of CT to evaluate the percentage of myocardial ischemia strengthens its potential to become the truly "one-stop shop" in CAD diagnostics.
We showed that VOL CTP is superior to the traditional CTP, coronary CTA, and CT-FFR in discriminating patients requiring coronary revascularization. Our results also underline the importance of MBF quantification for the appropriate planning of interventional therapies in chronic coronary syndromes. The newly developed VOL CTP uniquely combined the highest sensitivities and specificities in selecting patients for coronary revascularization, successfully sorting out the patients with a small ischemic area that can be successfully treated medically. This may save a significant proportion of patients from unnecessary invasive interrogation and therapies. The results of our study may upgrade the indications for CTP examination over the previously postulated ones in the case of suboptimal coronary CTA diagnostic results (e.g., diffuse calcifications, motion artifacts). VOL CTP should also be considered in the case of an uncertain CT-FFR, such as borderline CT-FFR, the case where the threshold value $(\leq 0.80)$ is reached in the distal vessel, non-proximal stenosis, or stenosis in a relatively small vessel [12-14]. The more accurate designation of patients who may benefit from revascularization will likely be advantageous. VOL CTP-guided patient management allows for a more appropriate qualification for invasive therapies, which can prevent unnecessary revascularizations and enable earlier treatment of patients with probable future events. The prediction of not only elective but also total revascularizations through VOL CTP suggests that the method may be more 
sensitive to ischemia already present during the baseline examination but overlooked while using other methods.

We advance the utility of CTP examination and propose an optimal clinical context concordant with the current guidelines $[8,15,16]$. Our study attempted to develop a method of volumetric myocardial ischemia evaluation in dynamic CTP using the myocardial blood flow threshold. Previously, Kwon et al. [17] calculated an ischemic percentage based on the visual analysis of the myocardial attenuation. Our findings were validated by clinical sequelae of revascularization. When performing VOL CTP analysis, we can also indicate the ischemia localization, like CTP standard analysis. Manual volumetric analysis based on the index-MBF allows precise assessment of the true rather than only the estimated percentage of myocardial ischemia (based on the number of affected segments). Moreover, index-MBF allows the normalization of the inter-individual differences in maximal MBF previously described in some studies $[5,18,19]$.

There have been some studies that investigated the prognostic value of dynamic CTP, but none of them have analyzed volumetric ischemia assessment [20-25]. Tanabe et al. [7], in a small study (39 patients), investigated the expected MBF from Voronoi-diagram-based myocardial segmentation but did not evaluate the true percentage of myocardial ischemia. As shown in the meta-analysis conducted by Pontone et al., positive stress myocardial CTP added to coronary CTA has a very high diagnostic performance in identifying a functionally significant coronary lesion in a patient-based model [26]. The addition of dynamic CTP significantly improved the diagnostic performance of CTA in detecting functionally significant $C A D$, and it was shown to be comparable to the addition of CT-FFR [27]. The study by van Assen et al. [25] showed that dynamic CTP alone (using index-MBF defined as the ratio between the territory MBF and the global MBF) has a higher prognostic value for the prediction of major adverse cardiac events (including elective revascularization) than CTA and CT-FFR, independently of clinical risk factors. There have also been some recently published studies that compared CTP with CT-FFR [4, 5, 27, 28]. Baggiano et al. [28] recently reported that the addition of both CT-FFR and CTP to CTA in their study led to the reclassification of approximately one-third of the patients with an intermediate to high likelihood of CAD. Also, Pontone et al. [27] showed that the addition of dynamic CTP to CTA and CT-FFR in their study provided additional diagnostic accuracy with acceptable radiation exposure. The CTA+CTP strategy showed better performance than the CTA+CT-FFR strategy in ultimate therapeutic decision-making and choice of target vessels [28]. In contrast, Yang et al. [4] showed in their study that there was no significant difference between the area under the curve values of CT-FFR and CTP but that the diagnostic performance of CTA was improved by combining it with either CT-FFR or CTP. Also, Coenen et al. [5] showed that both
CTP and CT-FFR can identify functionally significant CAD, with comparable accuracy. These are consistent with our results as we showed that the CTP standard approach has a similar diagnostic value (AUC) as CT-FFR for elective- and total-revascularization prediction.

\section{Limitations}

Our study was a single-center investigation with a limited sample size. Despite this, however, we were able to identify highly significant differences between the investigated methods and illustrate the incremental value of VOL CTP. However, multi-center studies assessing dynamic CTP with more definite endpoints, such as death or myocardial infarction during a long-term follow-up, would strengthen our findings. We did not perform additional ECG/echocardiography examinations during the follow-up. No deaths occurred in our study during the follow-up. Importantly, the low radiation dose reported during the examination should not be automatically assumed to be available for other CT systems capable of performing dynamic CTP.

Special attention is necessary for three-vessel CAD due to the possible lower global MBF in the case of the functional significance of all the three stenosed vessels. In our study, none of the patients had three-vessel CAD. This represents a possible bias as ischemia determination by CTP is less accurate in multivessel disease (e.g. partial overlap of perfusion defects or balanced ischemia). Therefore, the high accuracy values may not be representative of the true performance of VOL CTP in unselected real-world patients.

However, we did not detect a mismatch between ischemia localization and an obstructive vessel territory. The applicability of CT-FFR and dynamic CTP may differ based on their advantages and disadvantages. CT-FFR cannot be used to evaluate coronary stenosis in all CTA results [29]. In our study, CT-FFR was not performed in three patients who had heavy calcifications. Moreover, VOL CTP cut-off $\geq 10 \%$ should not be used as a basis for decision-making regarding invasive therapies until confirmed by larger trial data.

\section{CONCLUSION}

VOL CTP assessment is feasible and can provide incremental value to coronary artery stenosis assessment via CTA and ischemia evaluation via CT-FFR or the standard quantitative CTP for the prediction of coronary revascularization.

\section{Supplementary material}

Supplementary material is available at https://journals. viamedica.pl/kardiologia_polska.

\section{Article information}

Funding: The study is funded by the National Science Center, Poland - grant no. 2015/19/B/NZ5/03502 (to CK) and supported by National Institute of Cardiology, Warsaw, Poland - project no. 4.21/III/19 (to AO).

Conflict of interest: $\mathrm{KN}$ reports unrestricted research support from Siemens Healthineers, Bayer, HeartFlow Inc. unrelated to the present work; consulting for Siemens Medical Solutions USA. Other authors do not have a conflict of interest regarding this work. 
Open access: This article is available in open access under Creative Common Attribution-Non-Commercial-No Derivatives 4.0 International (CC BY-NC-ND 4.0) license, allowing to download articles and share them with others as long as they credit the authors and the publisher, but without permission to change them in any way or use them commercially. For commercial use, please contact the journal office at kardiologiapolska@ptkardio.pl.

\section{REFERENCES}

1. Knuuti J, Wijns W, Saraste A, et al. 2019 ESC Guidelines for the diagnosis and management of chronic coronary syndromes. Eur Heart J. 2020;41(3): 407-477, doi: 10.1093/eurheartj/ehz425, indexed in Pubmed: 31504439.

2. Danad I, Szymonifka J, Twisk JWR, et al. Diagnostic performance of cardiac imaging methods to diagnose ischaemia-causing coronary artery disease when directly compared with fractional flow reserve as a reference standard: a meta-analysis. Eur Heart J. 2017; 38(13): 991-998, doi: 10.1093/eurheartj/ehw095, indexed in Pubmed: 27141095.

3. Seitun S, Clemente A, De Lorenzi C, et al. Cardiac CT perfusion and FFR: pathophysiological features in ischemic heart disease. Cardiovasc Diagn Ther. 2020; 10(6): 1954-1978, doi: 10.21037/cdt-20-414, indexed in Pubmed: 33381437.

4. Yang DH, Kim YH, Roh JH, et al. Diagnostic performance of on-site CT-derived fractional flow reserve versus $\mathrm{CT}$ perfusion. Eur Heart J Cardiovasc Imaging. 2017; 18(4): 432-440, doi: 10.1093/ehjci/jew094, indexed in Pubmed: 27354345.

5. Coenen A, Rossi A, Lubbers MM, et al. Integrating CT myocardial perfusion and CT-FFR in the work-up of coronary artery disease. JACC CardiovasC Imaging. 2017; 10(7): 760-770, doi: 10.1016/j.jcmg.2016.09.028, indexed in Pubmed: 28109933.

6. Neumann FJ, Sousa-Uva M, Ahlsson A, et al.2018ESC/EACTS Guidelines on myocardial revascularization. Eurolntervention. 2019; 14(14): 1435-1534, doi: 10.4244/eijy19m01_01, indexed in Pubmed: 30667361.

7. Tanabe Y, Kido T, Kurata A, et al. Combined assessment of subtended myocardial volume and myocardial blood flow for diagnosis of obstructive coronary artery disease using cardiac computed tomography: A feasibility study. J Cardiol. 2020; 76(3): 259-265, doi: 10.1016/j.jjcc.2020.03.006, indexed in Pubmed: 32359811.

8. Oleksiak A, Kruk M, Pugliese F, et al. Regadenoson dynamic computed tomography myocardial perfusion using low-dose protocol for evaluation of the ischemic burden. ULYSSES study. J Cardiovasc Comput Tomogr. 2020; 14(5): 428-436, doi: 10.1016/j.jcct.2020.01.004, indexed in Pubmed: 32029383.

9. Thygesen $\mathrm{K}$, Alpert J, Jaffe $\mathrm{A}$, et al. Fourth universal definition of myocardial infarction (2018). Circulation. 2018; 138(20), doi: 10.1161/cir.0000000000000617, indexed in Pubmed: 30571511.

10. Raff GL, Abidov A, Achenbach S, et al. SCCT guidelines for the interpretation and reporting of coronary computed tomographic angiography. J Cardiovasc Comput Tomogr. 2009; 3(2): 122-136, doi: 10.1016/j. jcct.2009.01.001, indexed in Pubmed: 19272853.

11. Renker M, Schoepf UJ, Wang R, et al. Comparison of diagnostic value of a novel noninvasive coronary computed tomography angiography method versus standard coronary angiography for assessing fractional flow reserve. Am J Cardiol. 2014; 114(9): 1303-1308, doi: 10.1016/j.amjcard.2014.07.064, indexed in Pubmed: 25205628.

12. KrukM, WardziakŁ, Demkow M, etal. Workstation-Based calculation of CTAbased FFR for intermediate stenosis. JACC Cardiovasc Imaging. 2016; 9(6): 690-699, doi: 10.1016/j.jcmg.2015.09.019, indexed in Pubmed: 26897667.

13. Solecki M, Kruk M, Demkow M, et al. What is the optimal anatomic location for coronary artery pressure measurement at CT-derived FFR? J CardiovasC Comput Tomogr. 2017; 11(5): 397-403, doi: 10.1016/j.jcct.2017.08.004, indexed in Pubmed: 28844869.

14. Kruk M, Demkow M, Solecki $M$, et al. The location of distal coronary artery pressure measurement matters for computed tomography-derived fractional flow reserve. JACC Cardiovasc Imaging. 2018; 11 (2 Pt 1): 284-285, doi: 10.1016/j.jcmg.2017.11.023, indexed in Pubmed: 29413647.

15. Oleksiak A, Kruk M, Śpiewak M, et al. Safety of regadenoson with theophylline reversal during dynamic computed tomography perfusion and magnetic resonance imaging in patients with coronary artery disease. Kardiol Pol. 2020; 78(7-8): 709-714, doi: 10.33963/KP.15356, indexed in Pubmed: 32395975.

16. Oleksiak A, Sobieszczańska-Małek M, KrukM, et al. Feasibility of computed tomography perfusion for detection of cardiac allograft rejection following heart transplantation. JACC Cardiovasc Imaging. 2020; 13(5): 12861289, doi: 10.1016/j.jcmg.2020.01.004, indexed in Pubmed: 32061561.

17. Kwon $\mathrm{O}, \mathrm{Hwang} \mathrm{HJ}, \mathrm{Koo} \mathrm{HJ}$, et al. Ischemic burden assessment of myocardial perfusion CT, compared with SPECT using semi-quantitative and quantitative approaches. Int J Cardiol. 2019; 278: 287-294, doi: 10.1016/j. ijcard.2018.12.046, indexed in Pubmed: 30587418.

18. Kono AK, Coenen A, Lubbers M, et al. Relative myocardial blood flow by dynamic computed tomographic perfusion imaging predicts hemodynamic significance of coronary stenosis better than absolute blood flow. Invest Radiol. 2014;49(12):801-807, doi: 10.1097/RLI.0000000000000087, indexed in Pubmed: 25014013.

19. Rossi A, Wragg A, Klotz E, et al. Dynamic Computed Tomography Myocardial Perfusion Imaging: Comparison of Clinical Analysis Methods for the Detection of Vessel-Specific Ischemia. Circ Cardiovasc Imaging. 2017; 10(4), doi: 10.1161/CIRCIMAGING.116.005505, indexed in Pubmed: 28389506.

20. Pontone G, Andreini D, Guaricci Al, et al. Incremental diagnostic value of stress computed tomography myocardial perfusion with whole-heart coverage CT scanner in intermediate- to high-risk symptomatic patients suspected of coronary artery disease. JACC Cardiovasc Imaging. 2019; 12(2): 338-349, doi: 10.1016/j.jcmg.2017.10.025, indexed in Pubmed: 29454774.

21. Nakamura S, Kitagawa K, Goto $Y$, et al. Incremental prognostic value of myocardial blood flow quantified with stress dynamic computed tomography perfusion imaging. JACC Cardiovasc Imaging. 2019; 12(7 Pt 2): 1379-1387, doi: 10.1016/j.jcmg.2018.05.021, indexed in Pubmed: 30031698.

22. Nakamura S, Kitagawa K, Goto Y, et al. Prognostic value of stress dynamic computed tomography perfusion with computed tomography delayed enhancement. JACC Cardiovasc Imaging. 2020; 13(8): 1721-1734, doi: 10.1016/j.jcmg.2019.12.017, indexed in Pubmed: 32061554.

23. Meinel FG, Pugliese F, Schoepf UJ, et al. Prognostic value of stress dynamic myocardial perfusion $\mathrm{CT}$ in a multicenter population with known or suspected coronary artery disease. AJR Am J Roentgenol. 2017; 208(4): 761-769, doi: 10.2214/AJR.16.16186, indexed in Pubmed: 28177653.

24. Meinel FG, Wichmann JL, Schoepf UJ, et al. Global quantification of left ventricular myocardial perfusion at dynamic CT imaging: Prognostic value. J Cardiovasc Comput Tomogr. 2017; 11(1): 16-24, doi: 10.1016/j. jcct.2016.12.003, indexed in Pubmed: 28111212.

25. van Assen M, De Cecco CN, Eid M, et al. Prognostic value of CT myocardial perfusion imaging and $\mathrm{CT}$-derived fractional flow reserve for major adverse cardiac events in patients with coronary artery disease. J Cardiovasc Comput Tomogr. 2019; 13(3): 26-33, doi: 10.1016/j.jcct.2019.02.005, indexed in Pubmed: 30796003.

26. Pontone G, Guaricci Al, Palmer SC, et al. Diagnostic performance of non-invasive imaging for stable coronary artery disease: A meta-analysis. Int J Cardiol. 2020; 300: 276-281, doi: 10.1016/j.ijcard.2019.10.046, indexed in Pubmed: 31748186.

27. Pontone G, Baggiano A, Andreini D, et al. Dynamic stress computed tomography perfusion with a whole-heart coverage scanner in addition to coronary computed tomography angiography and fractional flow reserve computed tomography derived. JACC Cardiovasc Imaging. 2019; 12(12): 2460-2471, doi: 10.1016/j.jcmg.2019.02.015, indexed in Pubmed: 31005531.

28. Baggiano A, Fusini L, Del Torto A, et al. Sequential Strategy Including FFR Plus Stress-CTP Impacts on Management of Patients with Stable Chest Pain: The Stress-CTP RIPCORD Study. J Clin Med. 2020; 9(7), doi: 10.3390/jcm9072147, indexed in Pubmed: 32650379.

29. Driessen RS, Danad I, Stuijfzand WJ, et al. Comparison of coronary computed tomography angiography, fractional flow reserve, and perfusion imaging for ischemia diagnosis. J Am Coll Cardiol. 2019; 73(2): 161-173, doi: 10.1016/j.jacc.2018.10.056, indexed in Pubmed: 30654888. 